\title{
Erratum
}

\section{Erratum to "NCCAM/NCI Phase 1 Study of Mistletoe Extract and Gemcitabine in Patients with Advanced Solid Tumors"}

\author{
Patrick J. Mansky, ${ }^{1,2}$ Dawn B. Wallerstedt, ${ }^{3}$ Timothy S. Sannes, ${ }^{4}$ \\ Jamie Stagl, ${ }^{5}$ Laura Lee Johnson, ${ }^{2}$ Marc R. Blackman, ${ }^{6}$ Jean L. Grem, \\ Sandra M. Swain, ${ }^{8}$ and Brian P. Monahan' \\ ${ }^{1}$ The Cancer Team at Bellin Health, 1580 Commanche Avenue, Green Bay, WI 54313, USA \\ ${ }^{2}$ National Center for Complementary and Alternative Medicine, NIH, Bethesda, MD 20892, USA \\ ${ }^{3}$ Samueli Institute, Alexandria, VA 22314, USA \\ ${ }^{4}$ Department of Clinical and Health Psychology, University of Florida, Gainesville, FL 32611, USA \\ ${ }^{5}$ University of Miami, Miami, FL 33124, USA \\ ${ }^{6}$ Research Service (151), Veterans Affairs Medical Center and Department of Medicine, Georgetown University School of Medicine, \\ Washington, DC 20422, USA \\ ${ }^{7}$ University of Nebraska Medical Center, Omaha, NE 68198, USA \\ ${ }^{8}$ Washington Cancer Institute, Washington Hospital Center, Washington, DC 20010, USA \\ ${ }^{9}$ Department of Medicine, Hematology and Medical Oncology Division, Uniformed Services University of the Health Sciences, \\ Bethesda, MD 20814, USA
}

Correspondence should be addressed to Patrick J. Mansky; manpaj@bellin.org

Received 22 November 2013; Accepted 18 December 2013; Published 12 February 2014

Copyright (C) 2014 Patrick J. Mansky et al. This is an open access article distributed under the Creative Commons Attribution License, which permits unrestricted use, distribution, and reproduction in any medium, provided the original work is properly cited.

There were several modifications in the paper. They are shown as follows.

Page 1, Abstract: the sentence in the abstract "Dose limiting toxicities (DLT) were grade (G) 3 nonhematologic and G4 hematologic events" should read "... and G4 hematologic events related to platelets and granulocytes only."

"GEM $1380 \mathrm{mg} / \mathrm{m}^{2}$ and mistletoe $250 \mathrm{mg}$ combined were the MTD" should read "GEM $1300 \mathrm{mg} / \mathrm{m}^{2}$ and mistletoe $250 \mathrm{mg}$ combined were the MTD."

Page 2, Introduction: "While ML antibodies were absent in patients without adverse effects, ..." should read "While ML antibodies type IgE were absent in patients...."

Page 2, Materials and Methods: "A whole plant mistletoe extract (HELIXOR Apis (A), growing on fir trees)" should read "A whole plant mistletoe extract (Helixor A (Abietis), growing on fir trees)."

Page 2, Materials and Methods: "Helixor, GmbH, Rosenfeld" should read "Helixor Heilmittel GmbH \& Co. KG, Rosenfeld."
Page 3, Study Design and Outcomes: the sentence "... was administered with gemcitabine in $20 \%$ dose increments per dose level $\left(900,1080,1380\right.$, and $1560 \mathrm{mg} / \mathrm{m}^{2} \ldots$..)" should read “... (900, 1080, 1300, and $\left.1560 \mathrm{mg} / \mathrm{m}^{2} \ldots\right)$.."

Page 4, Results: Maximum Tolerated Dose and Dose Limiting Toxicities. The last sentence in this paragraph should read as follows: Thus, we achieved the MTD at dose level 8 (gemcitabine $1300 \mathrm{mg} / \mathrm{m}^{2}$ and mistletoe $250 \mathrm{mg}$ ).

Paragraph 3.4. "Three study participants experienced individual DLTs at dose level 9 (gemcitabine $1560 \mathrm{mg} / \mathrm{m}^{2}$ with $250 \mathrm{mg}$ daily of mistletoe). These included grade 3 cellulitis at the mistletoe injection site, grade 4 acute renal failure, and grade 4 neutropenia" should read "... and grade 4 thrombocytopenia."

Page 8, Discussion: the sentence "The MTD for the gemcitabine/mistletoe combination in this study was gemcitabine $1380 \mathrm{mg} / \mathrm{m}^{2}$..." should read "The MTD for the gemcitabine/mistletoe combination in this study was gemcitabine $1300 \mathrm{mg} / \mathrm{m}^{2} \ldots . . ”$ 
TABle 4: Dose limiting toxicities by dose level.

\begin{tabular}{|c|c|c|c|c|c|c|}
\hline \multicolumn{3}{|c|}{ Stage I (fixed GEM dose of $750 \mathrm{mg} / \mathrm{m}^{2}$ ) } & \multicolumn{4}{|c|}{ Stage II (fixed mistletoe dose, established in stage 1) } \\
\hline Level & $N$ & DLT & Level/dosage & $N$ & DLT & $\begin{array}{l}\text { Action taken per } \\
\text { protocol }\end{array}$ \\
\hline $\begin{array}{l}\text { (1) } \\
\text { (escalating daily } \\
\text { mistletoe injections, } \\
\text { reaching: } 20 \mathrm{mg} / \text { day) }\end{array}$ & 3 & None & $\begin{array}{c}\text { (6) } \\
(250 \mathrm{mg} / \text { day mistletoe; } \\
900 \mathrm{mg} / \mathrm{m}^{2} \text { GEM on } \\
\text { day } 1 / 8 \text { of } 3 \text {-week cycle })\end{array}$ & 7 & Grade 4 neutropenia & $\begin{array}{c}\text { Dose reduced; enrolled } 3 \\
\text { more patients at this } \\
\text { dose level. }\end{array}$ \\
\hline $\begin{array}{l}\text { (2) } \\
\text { (escalating daily } \\
\text { mistletoe injections, } \\
\text { reaching: } 50 \mathrm{mg} / \text { day) }\end{array}$ & 3 & None & $\begin{array}{c}(7) \\
(250 \mathrm{mg} / \text { day mistletoe; } \\
\mathbf{1 0 8 0} \mathrm{mg} / \mathrm{m}^{2} \text { GEM on } \\
\text { day } 1 / 8 \text { of } 3 \text {-week cycle })\end{array}$ & 7 & $\begin{array}{c}\text { Grade } 4 \\
\text { thrombocytopenia }\end{array}$ & $\begin{array}{c}\text { Dose reduced; enrolled } 3 \\
\text { more patients at this } \\
\text { dose level. }\end{array}$ \\
\hline $\begin{array}{l}\text { (3) } \\
\text { (escalating daily } \\
\text { mistletoe injections, } \\
\text { reaching: } 100 \mathrm{mg} / \text { day) }\end{array}$ & 3 & None & 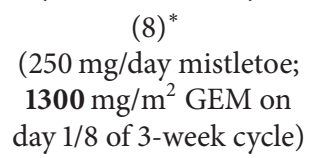 & 6 & None & N/A \\
\hline $\begin{array}{l}\text { (escalating daily } \\
\text { mistletoe injections, } \\
\text { reaching: } 200 \mathrm{mg} / \text { day) }\end{array}$ & 6 & None & $\begin{array}{c}(9) \\
(250 \mathrm{mg} / \text { day mistletoe; } \\
\mathbf{1 5 6 0} \mathrm{mg} / \mathrm{m}^{2} \text { GEM on } \\
\text { day } 1 / 8 \text { of } 3 \text {-week cycle })\end{array}$ & 4 & $\begin{array}{l}\text { Grade } 3 \text { cellulitis }^{\mathrm{a}} \text {; } \\
\text { grade } 4 \text { acute renal } \\
\text { failure }^{\mathrm{b}} \text {; grade } 4 \text { : } \\
\text { thrombocytopenia }^{\mathrm{c}}\end{array}$ & $\begin{array}{l}{ }^{\text {a }} \text { mistletoe therapy } \\
\text { withheld; patient } \\
\text { rechallenged and } \\
\text { developed } \\
\text { hypersensitivity } \\
\text { reaction. Mistletoe } \\
\text { discontinued; }{ }^{b} \mathrm{Pt} \text {. } \\
\text { treated for renal failure } \\
\text { and subsequently } \\
\text { withdrawn from study; } \\
{ }^{\mathrm{c}} \mathrm{Pt} \text { hospitalized d/t } \\
\text { other AE; Maximum } \\
\text { tolerated dose reached } \\
\text { per protocol. }\end{array}$ \\
\hline $\begin{array}{l}\text { (5) } \\
\text { (escalating daily } \\
\text { mistletoe injections, } \\
\text { reaching: } 250 \mathrm{mg} / \text { day) }\end{array}$ & 5 & None & & & & \\
\hline
\end{tabular}

${ }^{*}$ Per study protocol, this level represents the maximum tolerated dose, as 3 DLT's were observed in the subsequent dose level.

Page 8, Table 4: Dose limiting toxicities by dose level. "Level/dosage 8 (250 mg/day mistletoe; $1380 \mathrm{mg} / \mathrm{m}^{2} \mathrm{GEM}$ on day $1 / 8$ of 3 -week cycle)" should read "Level/dosage 8 ( $250 \mathrm{mg} /$ day mistletoe; $1300 \mathrm{mg} / \mathrm{m}^{2} \mathrm{GEM}$ on day $1 / 8 \mathrm{of} 3-$ week cycle)."

Page 9, Discussion: the sentence "None of the study patients developed febrile neutropenia even at the highest gemcitabine dose of $1650 \mathrm{mg} / \mathrm{m}^{2}$ " should read "None of the study patients developed febrile neutropenia even at the highest gemcitabine dose of $1560 \mathrm{mg} / \mathrm{m}^{2}$."

Page 9, Conclusion: the sentence "the MTD was gemcitabine $1380 \mathrm{mg} / \mathrm{m}^{2}$ weekly ..." should read "the MTD was gemcitabine $1300 \mathrm{mg} / \mathrm{m}^{2}$ weekly ...."

Page 10, Acknowledgments: the second sentence should read: They greatly appreciate ... the support of Drs. Dietrich Schlodder and Jörg Schierholz.

The sentence “... as well as Sabine Rieger from Helixor GmbH” should read “. . . as well as Sabine Rieger from Helixor Heilmittel GmbH \& Co. KG."

The sentence "Mistletoe extract (Helixor A) was provided by Helixor GmbH, Rosenfeld, ..." should read "Mistletoe extract (Helixor A) was provided by Helixor Heilmittel GmbH \& Co. KG, Rosenfeld, ...." 


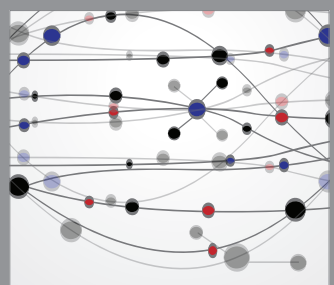

The Scientific World Journal
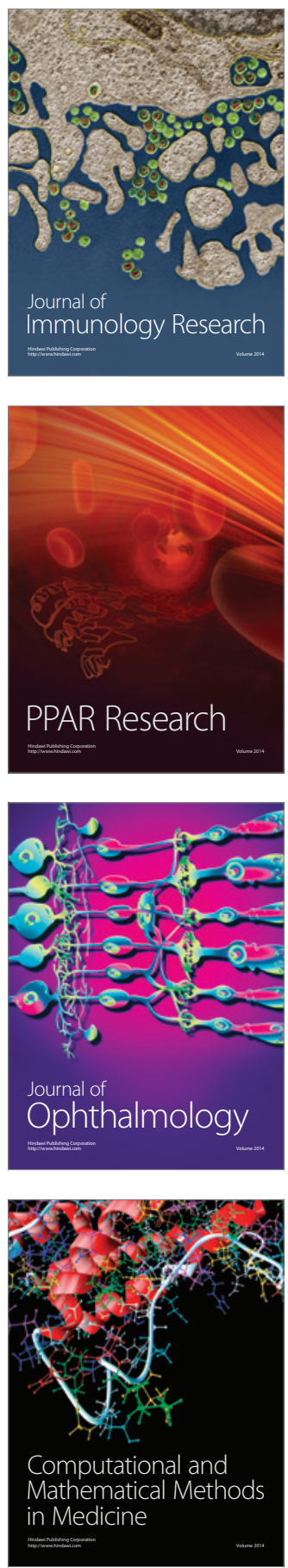

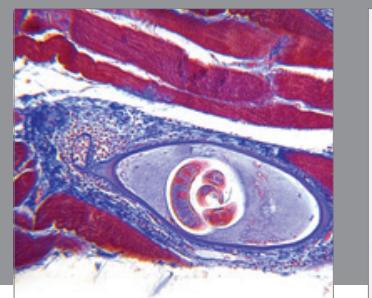

Gastroenterology

Research and Practice
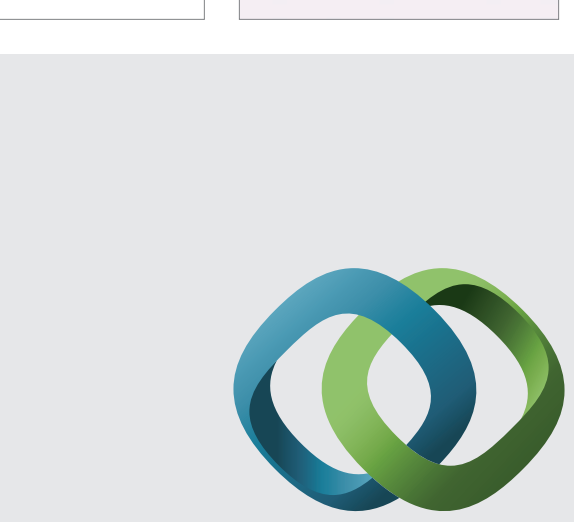

\section{Hindawi}

Submit your manuscripts at

http://www.hindawi.com
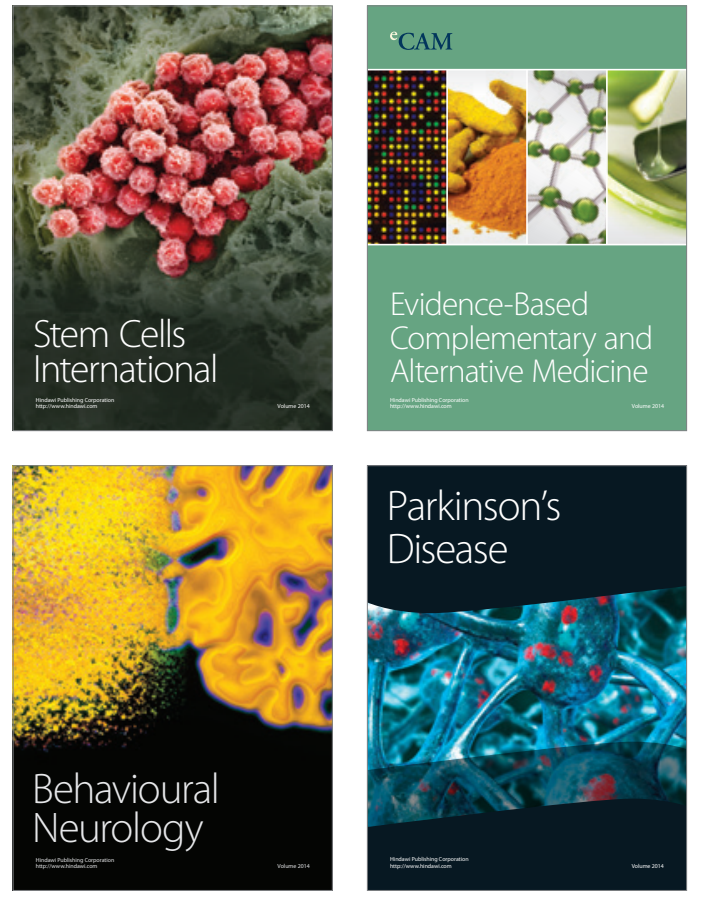
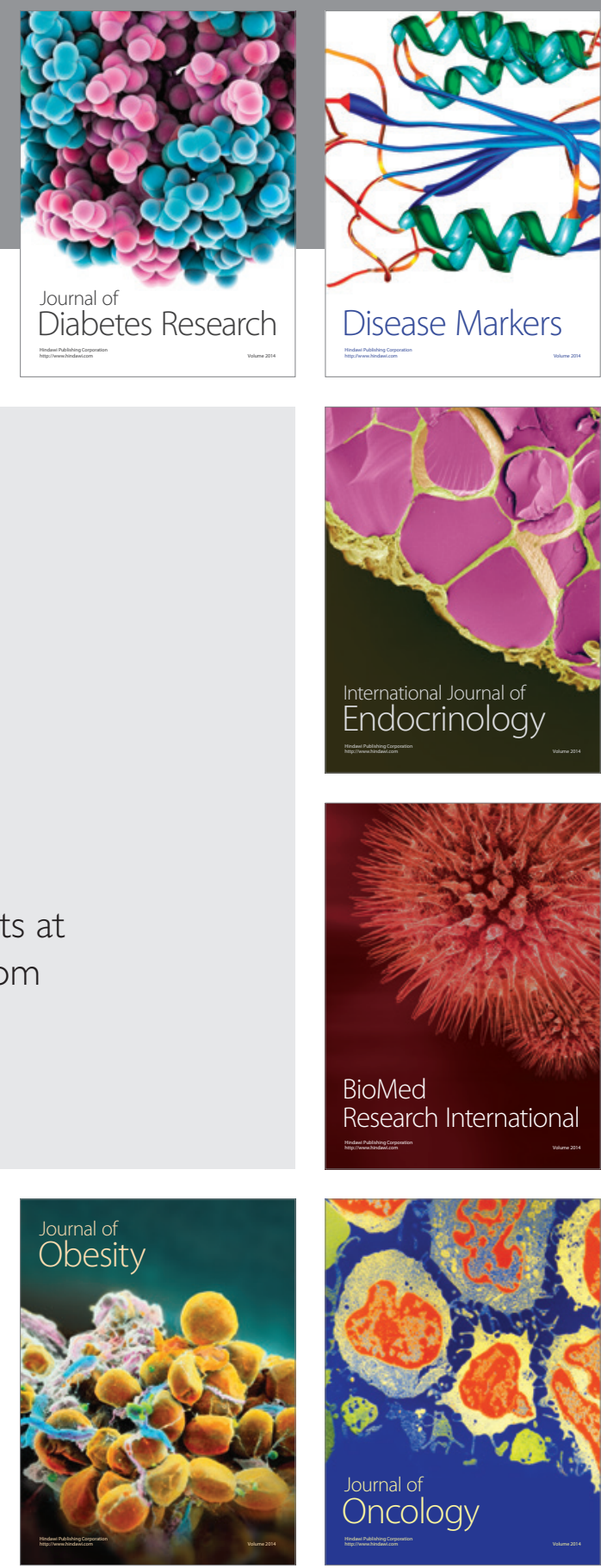

Disease Markers
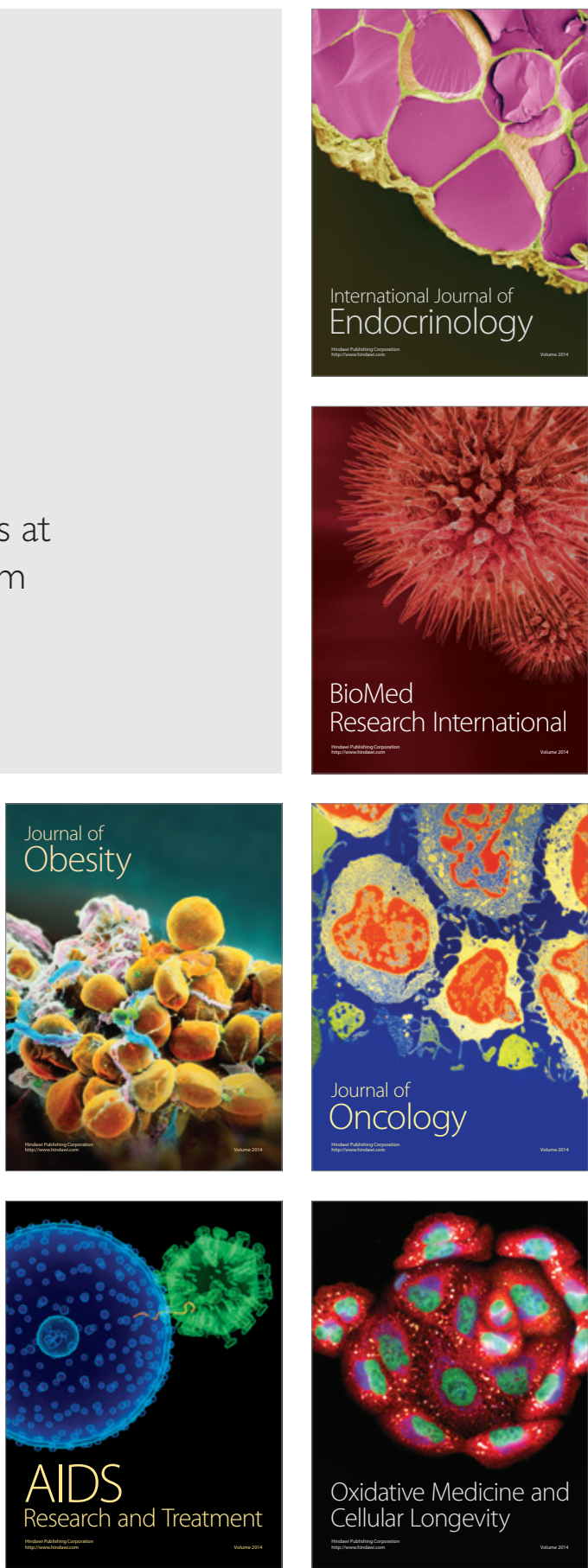